\title{
Epidemic features affecting the performance of outbreak detection algorithms
}

\author{
Jie Kuang ${ }^{1}$, Wei Zhong Yang ${ }^{2}$, Ding Lun Zhou', Zhong Jie Li ${ }^{2}$ and Ya Jia Lan ${ }^{1 *}$
}

\begin{abstract}
Background: Outbreak detection algorithms play an important role in effective automated surveillance. Although many algorithms have been designed to improve the performance of outbreak detection, few published studies have examined how epidemic features of infectious disease impact on the detection performance of algorithms. This study compared the performance of three outbreak detection algorithms stratified by epidemic features of infectious disease and examined the relationship between epidemic features and performance of outbreak detection algorithms.

Methods: Exponentially weighted moving average (EWMA), cumulative sum (CUSUM) and moving percentile method (MPM) algorithms were applied. We inserted simulated outbreaks into notifiable infectious disease data in China Infectious Disease Automated-alert and Response System (CIDARS), and compared the performance of the three algorithms with optimized parameters at a fixed false alarm rate of $5 \%$ classified by epidemic features of infectious disease. Multiple linear regression was adopted to analyse the relationship of the algorithms' sensitivity and timeliness with the epidemic features of infectious diseases.

Results: The MPM had better detection performance than EWMA and CUSUM through all simulated outbreaks, with or without stratification by epidemic features (incubation period, baseline counts and outbreak magnitude). The epidemic features were associated with both sensitivity and timeliness. Compared with long incubation, short incubation had lower probability $\left(\beta^{*}=-0.13, P<0.001\right)$ but needed shorter time to detect outbreaks $\left(\beta^{*}=-0.57\right.$, $P<0.001)$. Lower baseline counts were associated with higher probability $\left(\beta^{*}=-0.20, P<0.001\right)$ and longer time $\left(\beta^{*}=0.14, P<0.001\right)$. The larger outbreak magnitude was correlated with higher probability $\left(\beta^{*}=0.55, P<0.001\right)$ and shorter time $\left(\beta^{*}=-0.23, P<0.001\right)$.
\end{abstract}

Conclusions: The results of this study suggest that the MPM is a prior algorithm for outbreak detection and differences of epidemic features in detection performance should be considered in automatic surveillance practice.

Keywords: Epidemic feature, Outbreak detection algorithms, Performance, Automated infectious disease surveillance

\section{Background}

Infectious diseases remain the major causes of morbidity and mortality in China despite substantial progress in their control [1]. The outbreaks of infectious diseases pose serious threats on public health. Early detection of aberration and rapid control actions, which the Chinese Ministry of Health has taken as important strategies for

\footnotetext{
* Correspondence: lanyajia@sina.com

'Department of Occupational Health, West China School of Public Health, Sichuan University, 17 South Section 3 Renmin Road, Chengdu, Sichuan 610041, China

Full list of author information is available at the end of the article
}

emergency infectious disease prevention and control [2], are prerequisites for preventing the spread of outbreaks and reducing the morbidity and death caused by diseases. Therefore, China Infectious Disease Automated-alert and Response System (CIDARS) was successfully implemented and began to operate nationwide in 2008 [3].

At the end of 2010, analysis results of the operation of CIDARS in nationwide showed that a large number of outbreaks of infectious diseases could be timely detected, but it was also found that there were many of false-positive signals; large differences existed between outbreak signal counts and final identified outbreaks in different

\section{Biomed Central}


diseases; the detection performance was poor in those diseases which had more case reports and fewer outbreaks [4]. These issues prompted us that epidemic features of infectious disease may affect outbreak detection performance.

Several studies have described the determinants of outbreak detection performance, including: system factors (representativeness, outbreak detection algorithms and algorithm-specifics), outbreak characteristics (outbreak size, shape of the outbreak signal and time of the outbreak) [5-8]. Understanding the differences these determinants make in detection performance can help public health practitioners improve the automated surveillance system, thus raising detection capabilities. Recently, extensive researches have explored novel algorithms to improve the performance of outbreak detection [9-13], but evidence on how epidemic features impact on detection performance is still limited.

To address this limitation, our study aimed to explore the influence of epidemic features (incubation period, baseline counts and outbreak magnitude) on algorithms' detection performance. Findings of this study may help public health surveillance practitioners understand the detection performance of algorithms under these epidemic features and improve the implementation of automated surveillance.

\section{Methods}

Figure 1 presents the flow of data processing in this study. First, we selected eight notifiable infectious diseases in CIDARS for studying. Second, we sampled ten counties for each infectious disease. Third, we exacted the surveillance data sequences in 2005-2009 from CIDARS. Fourth, we marked the public health emergencies and injected simulated outbreaks in data sequences.
Fifth, we ran the three outbreak detection algorithms. Sixth, we computed the sensitivity and timeliness of the three outbreak detection algorithms. Finally, statistical inference and multiple linear regression were used to compare the performance and examine the relationship of sensitivity and timeliness with epidemic features.

\section{Data sources}

The infectious disease data were extracted from CIDARS. CIDARS was developed basing on the existing data from National Disease Surveillance Reporting and Management System on 28 diseases that are outbreakprone and require prompt action. The 28 diseases were classified into two types according to severity, incidence rate and importance [3]. Type 1 diseases includes nine infectious diseases characterized with higher severity but lower incidence and are analysed using fixed-threshold detection method. For type 2 diseases (19 more common infectious diseases), we selected eight diseases (dysentery, scarlet fever, mumps, measles, malaria, typhoid, encephalitis B and hepatitis A) which represented three routes of transmission(respiratory, oral-fecal and vectorborne). Five provinces were sampled for eight diseases (dysentery in Hunan, scarlet fever, measles in Xinjiang, mumps in Chongqing, malaria, typhoid, encephalitis B in Yunnan, hepatitis A in Guizhou), where the respective disease had high incidence and became important local public health problems. Then we randomly sampled 10 counties from the selected provinces for each disease, and obtained their actual daily number of reported cases in 2005-2009. Data from 2005 to 2007 were used as baseline, while data from 2008 to 2009 were used to evaluate the algorithms.

Strategy of inserting simulated outbreaks was used to evaluate the detection performance. To prevent public

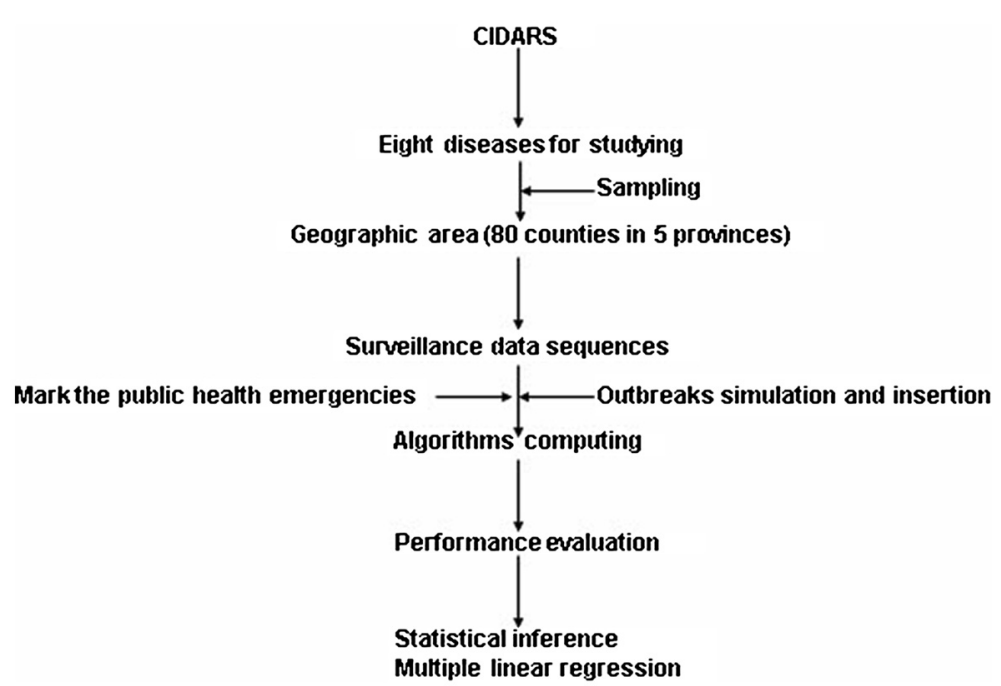

Figure 1 The flowchart of data processing. 
health emergency confounding the evaluation, we got the records of public health emergency in the corresponding counties from Emergency Public Reporting System [14].

\section{Outbreak detection algorithms}

To date many outbreak detection algorithms can be used for temporal data [15-19]. Considering that we collected five years data, using the same periods' historical data as baseline is appropriate, to some extent, can reduce the seasonal and day-of-week variation in the baseline. However, for using this, the regression and ARIMA models may subject to certain restrictions, as the steps in their processing require recent continuous time interval to calculate expected statistic. So we chose two most commonly used statistical process control algorithms (EWMA, CUSUM) and a non-parameter algorithm (MPM) which enable the application of the same periods' historical data in theory.

For EWMA, the current time-series value is replaced by a weighted average of the recent values. If the observed values were assumed for Poisson distribution, The smoothed daily count was calculated as

$$
Z_{t}=\lambda \bar{X}_{\mathrm{t}}+\cdot(1-\lambda) \cdot Z_{\mathrm{t}-1}
$$

and the upper control limit(UCL) was calculated as

$$
\mathrm{UCL}=\mu+\cdot \mathrm{k} \sigma \sqrt{\frac{\lambda}{2-\lambda}}
$$

In the algorithm, the $\lambda(0<\lambda<1)$ was the weighting factor, $\mathrm{k}$ was the control limit coefficient [20].

Cumulative Sum (CUSUM) has been used to detect outbreaks of infectious diseases in recent decades [21]. Two parameters $\mathrm{k}$ and $\mathrm{h}$ are involved. $\mathrm{k}$ refers to the pre-specified reference value and $h$ the decision boundary. Here, $\mu_{\mathrm{t}}$ is the sample mean and $\sigma_{\mathrm{t}}$ the standard deviation of the reference values. When we denote $x_{t}$ the count per day, then the CUSUM statistic is defined as

$$
\mathrm{S}_{\mathrm{t}}=\max \left(0, \mathrm{~S}_{\mathrm{t}-1}+\left(\mathrm{x}_{\mathrm{t}}-\left(\mu_{\mathrm{t}}+\mathrm{k} \sigma_{\mathrm{t}}\right) / \sigma_{\mathrm{t}}\right)\right)
$$

If the CUSUM statistic is larger than $h$, then the current day is considered as a possible outbreak.

The MPM uses previous several years (such as 35 years) over the same period as baseline data, setting a percentile of baseline data as a detection parameter $\mathrm{c}$. If the current day counts $x_{t}$ is greater than the detection parameter's corresponding percentile $\left(\mathrm{P}_{\mathrm{c}}\right)$, outbreak signal is produced [22].

\section{Algorithm parameters}

To obtain the optimized parameter values of the three algorithms, we used R software [23] generating two-year Poisson distribution sequences with five daily average

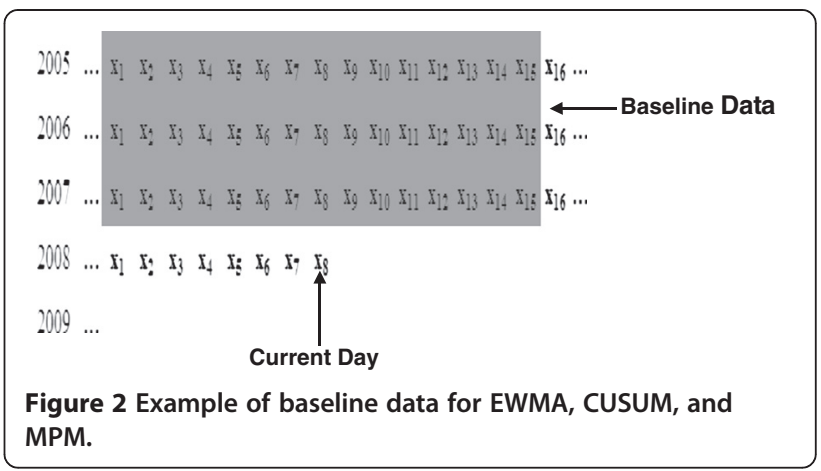

counts levels $(0.1,0.5,1.0,2.0,5.0)$. We set a fixed false alarm rate of 0.05 (an average of one false alarm every twenty days) by applying each algorithm to these five sequences without any added outbreak signals, and determining the parameters that would yield an average of one false alert every twenty days (see Additional file 1: Table S1).

\section{Baseline data}

We used baseline data from 2005 to 2007, in which we use the corresponding day, the seven previous days and the seven later days. These summed up to a total of 45 days (Figure 2). The EWMA algorithm was associated with the order day of baseline data (Figure 3).

\section{Outbreak simulation and insertion}

We generated outbreak signals by simulation. We selected real outbreak events reported in the literatures for each disease [24-31], and calculated the proportion of daily case distribution (Figure 4). Next, we simulated four outbreak magnitudes (0.5, 1.0, 2.0, 3.0), which meant that the increase cases of outbreak signals are the corresponding multiple of the baseline number of cases.

As the baselines were required to use the same period of history (seven days before and after the current day), to increase the stability of the calculation, we made 10th-day the insertion date of outbreak signals every month. If the intervals had public health emergency, then skipped the insertion. As the outbreak duration of






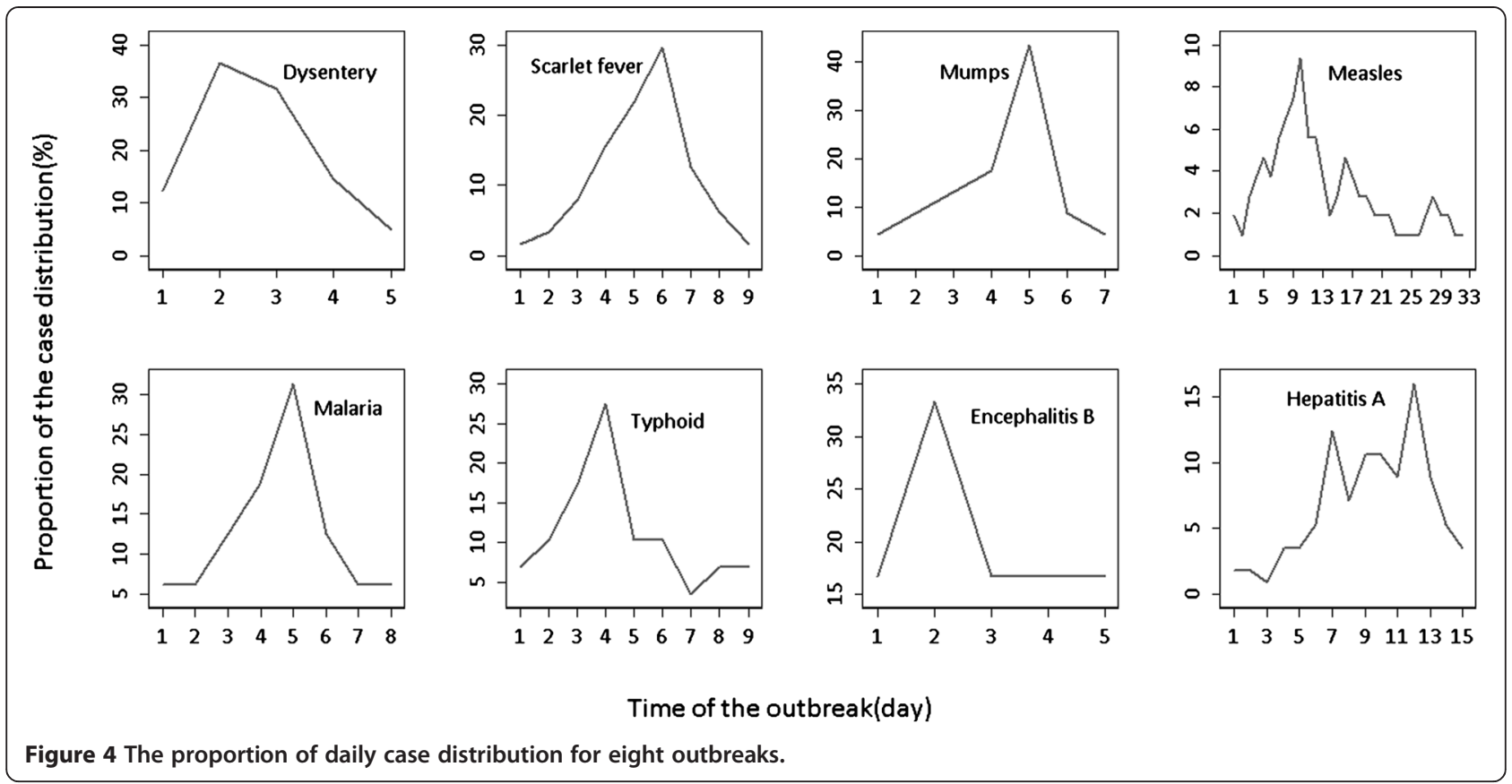

measles was more than one month, the outbreak signals were injected every two months. Finally, we had eight kinds of outbreak signals, each disease included ten counties, and each county had four outbreak magnitude test datasets, which summed up to 320 test datasets. In theory, 7,200 outbreak signals could be inserted. Excluding the public health emergencies, the actual outbreak signals inserted were 7,088 (Table 1).

\section{Incubation classification}

According to the minimum, maximum, average incubation period of eight diseases [32], eight diseases were clustered into three categories by K-means clustering method: the short incubation period disease (dysentery,

Table 1 Summary the number of injected outbreak signals

\begin{tabular}{lllll}
\hline Diseases & County & $\begin{array}{l}\text { Outbreaks } \\
\text { should be } \\
\text { injected }\end{array}$ & $\begin{array}{l}\text { Public } \\
\text { health } \\
\text { emergencies }\end{array}$ & $\begin{array}{l}\text { Actual } \\
\text { injected } \\
\text { outbreaks }\end{array}$ \\
\hline Dysentery & 10 & 960 & 1 & 956 \\
Scarlet fever & 10 & 960 & 3 & 948 \\
Mumps & 10 & 960 & 20 & 880 \\
Measles & 10 & 480 & 0 & 480 \\
Malaria & 10 & 960 & 0 & 960 \\
Typhoid & 10 & 960 & 1 & 956 \\
Encephalitis B & 10 & 960 & 3 & 948 \\
Hepatitis A & 10 & 960 & 0 & 960 \\
Total & 80 & 7200 & 28 & 7008 \\
\hline
\end{tabular}

scarlet fever), the medium incubation period disease (mumps, measles, malaria, typhoid, encephalitis B) and the long incubation period disease (hepatitis A) (Table 2).

\section{Performance evaluation}

Performance comparisons were based on three indicators: sensitivity (the proportion of outbreaks the algorithm detected), timeliness (the difference between the date of the first true alarm and the beginning date of the outbreak) and false alarm rate (the proportion of nonoutbreak days on which the algorithm signal an alarm).

For more informative comparisons of the performance in different determinants, we plotted sensitivity-timeliness curve [33], which measured the proportion of

Table 2 Classification the incubation of diseases with Kmeans clustering

\begin{tabular}{|c|c|c|c|c|}
\hline \multirow[t]{2}{*}{ Diseases } & \multicolumn{3}{|c|}{ Incubation period(day) } & \multirow{2}{*}{$\begin{array}{l}\text { Clustering } \\
\text { classification }\end{array}$} \\
\hline & Minimum & Maximum & Average & \\
\hline Dysentery & 1 & 7 & 2 & Short \\
\hline Scarlet fever & 2 & 12 & 4 & Short \\
\hline Mumps & 8 & 30 & 18 & Medium \\
\hline Measles & 6 & 21 & 10 & Medium \\
\hline Malaria & 7 & 30 & 17 & Medium \\
\hline Typhoid & 5 & 21 & 9 & Medium \\
\hline Encephalitis B & 10 & 15 & 12 & Medium \\
\hline Hepatitis A & 15 & 45 & 30 & Long \\
\hline
\end{tabular}


Table 3 Summary the performance of outbreak detection algorithms

\begin{tabular}{lllll}
\hline $\begin{array}{l}\text { Performance } \\
\text { indicators }\end{array}$ & Mean & $\begin{array}{l}\text { 95\% confidence } \\
\text { interval }\end{array}$ & F value & $\boldsymbol{P}$-value \\
\hline Sensitivity(\%) & & & & \\
EWMA & 56.02 & $53.08-58.95$ & 22.04 & $<0.001$ \\
CUSUM & 58.72 & $55.81-61.63$ & & \\
MPM* & 69.71 & $66.46-72.96$ & & \\
Timeliness(Day) & & & & \\
EWMA & 2.40 & $2.20-2.62$ & 20.28 & $<0.001$ \\
CUSUM & 2.52 & $2.31-2.73$ & & \\
MPM* & 1.65 & $1.45-1.85$ & & \\
False alarm rate(\%) & & & & \\
EWMA & 5.09 & $4.39-5.78$ & 1.88 & 0.153 \\
CUSUM & 5.85 & $5.08-6.63$ & & \\
MPM & 4.93 & $4.28-5.58$ & & \\
\hline P $<0.001$ According & $B 0 n f$. & & \\
\hline
\end{tabular}

${ }^{*} p<0.001$.According to Bonferroni's procedure, the family wise error rate was 0.05 , divided by the number of test. The significance level afor an individual test was $0.05 / 3$, being 0.017

outbreaks that an algorithm detected within several days from the start of the outbreak.

\section{Statistical inference and multiple linear regression}

For each of the eight diseases in each of the ten counties on each of the four out- break magnitudes, we computed the sensitivity, timeliness and false alarm rate across all 320 analysis runs. We used ANOVA to test for significant difference in the algorithms' sensitivities and timeliness. The Bonferroni correction was applied for multiple comparisons to control the family wise error rate. The significance level $\alpha$ was 0.05 . Finally, multiple linear regression was run to understand the relationship of the algorithms sensitivities and timeliness with disease incubation, baseline counts and outbreak magnitude. All the analyses were performed using R software.

\section{Results}

\section{Algorithm performance}

The respective proportion of detected outbreak signals of EWMA, CUSUM, MPM were 56.02\%, 58.72\%, 69.71\%. Overall, the MPM was more sensitive than EWMA, CUSUM. In timeliness, the average lag days of all outbreak signals were: EWMA 2.40, CUSUM 2.52 and MPM 1.65. Compared with EWMA and CUSUM, the MPM needed shorter time to detect outbreak signals ( $\mathrm{P}<0.001)$. The false alarm rate of EWMA, CUSUM and MPM were 5.09, 5.85 and 4.93. The false alarm rate did not differ in three algorithms $(\mathrm{P}=0.153)$ (Table 3$)$.

\section{Sensitivity-timeliness plot}

Figures 4, 5, 6 were sensitivity-timeliness plot to compare three algorithms performance in different incubation categories, baseline counts levels and outbreak magnitudes.

In the three incubation categories, the MPM had a higher probability of detecting the outbreak than CUSUM and EWMA within all days from the start of the outbreak; the sensitivity changes of CUSUM and EWMA over time were very close. In short incubation, the sensitivity of MPM reached $90 \%$ at the sixth day of a outbreaks start. The long incubation disease had a poorer sensitivity than short and medium incubation disease (Figure 5).

We used the 33.33, 66.67 percentiles with the cuts off $0.05,0.2$ to divide average baseline counts into low, medium and high levels. At the three levels, the MPM had a higher probability of detecting the outbreak than CUSUM and EWMA within all days from the start of the outbreak; the sensitivity of CUSUM and EWMA was very closely changed over time. The sensitivity of low baseline counts was better than medium and high levels, with the MPM reaching higher than 90\% (Figure 6).

In the four outbreak magnitudes, the MPM had a higher probability of detecting outbreak than CUSUM

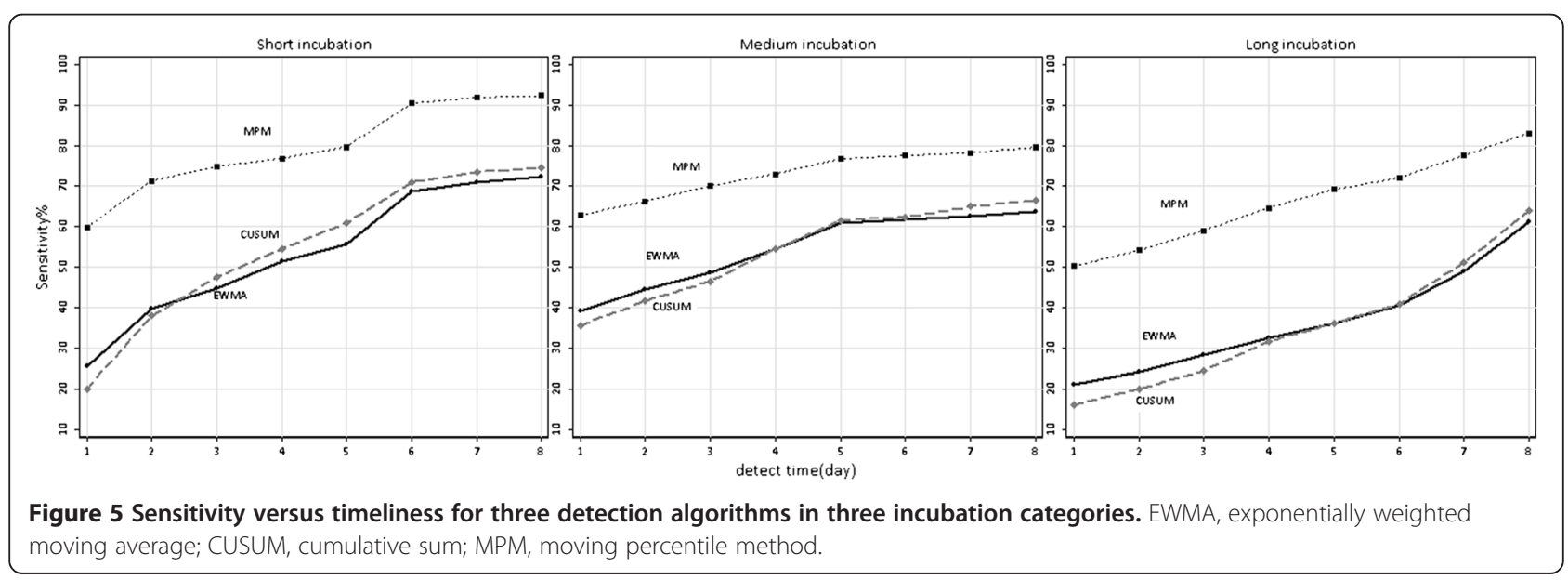




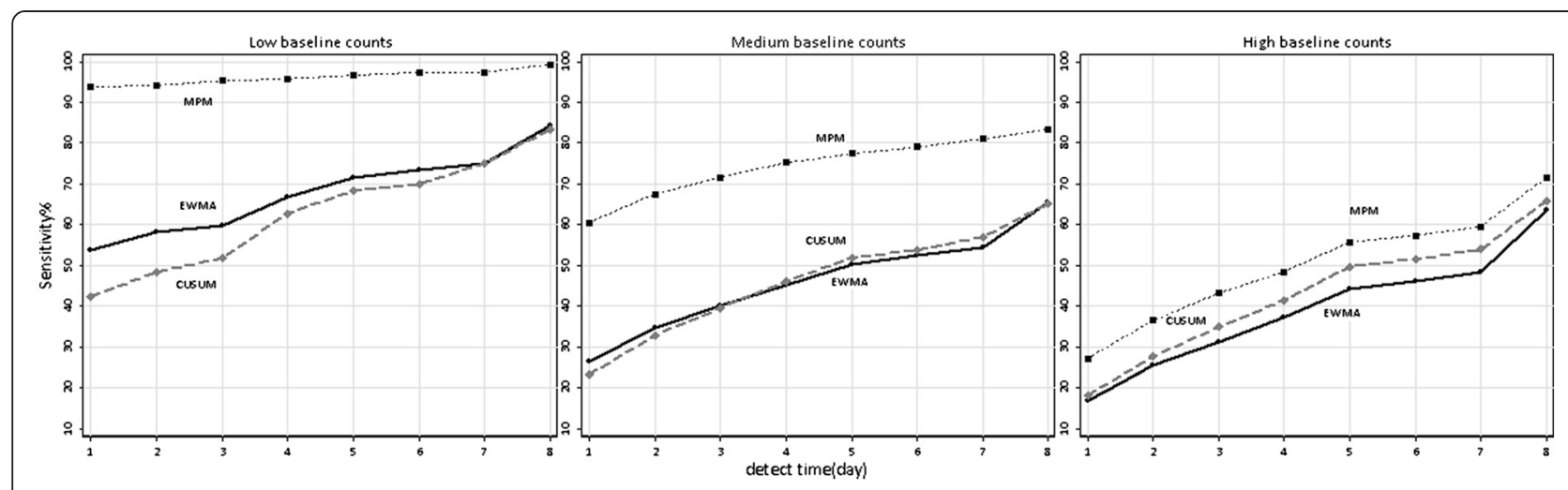

Figure 6 Sensitivity versus timeliness for three detection algorithms in three baseline counts levels. EWMA, exponentially weighted moving average; CUSUM, cumulative sum; MPM, moving percentile method.

and EWMA within all days from the start of the outbreak; the CUSUM method had a better sensitivity than EWMA in 2.0,3.0 magnitude (Figure 7).

\section{Multiple linear regression}

The variable coding in multiple linear regression (Table 4).

The results of multiple linear regression showed that algorithms, incubation period, baseline counts and outbreak magnitude were associated with both sensitivity (Table 5) and timeliness (Table 6).

As it was illustrated in Table 5, the algorithms, incubation period, baseline counts and outbreak magnitude had a statistically significant relationship with sensitivity. MPM had a higher probability of detecting outbreaks compared with EWMA $\left(\beta^{*}=0.21, P<0.001\right)$. Short $\left(\beta^{*}=-0.13, \mathrm{P}<0.001\right)$ and Medium $\left(\beta^{*}=-0.11, \mathrm{P}=0.002\right)$ incubation had a lower probability of detecting the outbreaks compared with long incubation. The lower the baseline counts, the higher probability $\left(\beta^{*}=-0.20, \mathrm{P}\right.$ $<0.001)$. The larger outbreak magnitude, the higher probability $\left(\beta^{*}=0.55, \mathrm{P}<0.001\right)$.
The algorithms, incubation period, baseline counts and outbreak magnitude had a statistically significant relationship with timeliness. MPM needed shorter time to detect the outbreaks compared with $\operatorname{EWMA}\left(\beta^{*}=-0.17\right.$, $P<0.001)$. Short $\left(\beta^{*}=-0.57, \quad P<0.001\right)$ and Medium $\left(\beta^{*}=-0.56, P<0.001\right)$ incubation needed shorter time to detect the outbreaks compared with long incubation. The higher baseline counts, the longer time to detect the outbreaks ( $\left.\beta^{*}=0.14, P<0.001\right)$. The larger outbreak magnitude, the shorter time $\left(\beta^{*}=-0.23, \quad P<0.001\right)$ (Table 6).

\section{Discussion}

Many determinants affect the performance of outbreak detection in automated surveillance, and knowing about how these factors influence the detection performance can help to improve automated surveillance system. In this study, we compared the performance of three outbreak detection methods by adding simulated outbreaks to actual daily counts of notifiable infectious diseases in CIDARS and examined the relationship of the detection

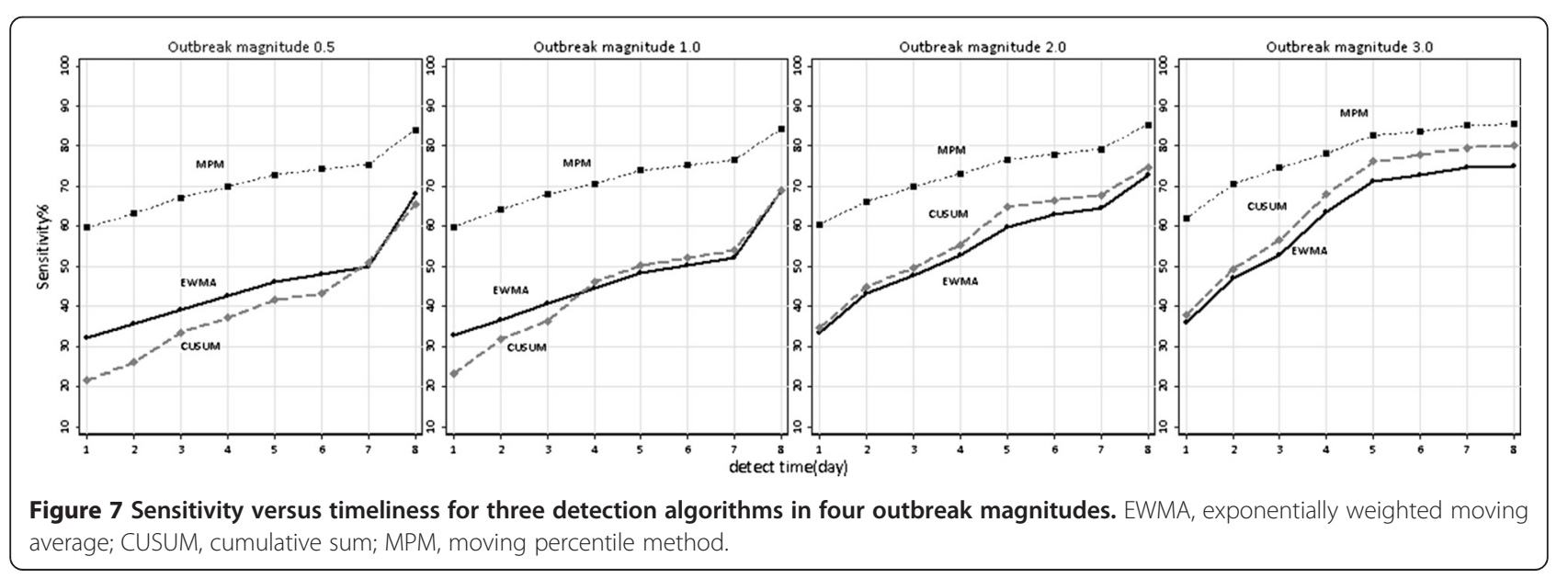


Table 4 Variable coding in multiple linear regression

\begin{tabular}{ll}
\hline Variable & Variable coding \\
\hline Dependent Variable & \\
Sensitivity or Timeliness & Actual value \\
Independent Variable & \\
Algorithm & 1 CUSUM \\
& $2 \mathrm{MPM}$ \\
& $3 \mathrm{EWMA}$ \\
Incubation & 1 Short \\
& 2 Medium \\
Baseline counts & 3 Long \\
Outbreak magnitude & Actual value \\
& 0.5 \\
& 1.0 \\
\hline
\end{tabular}

performance with disease incubation, baseline counts and outbreak magnitude.

In algorithms' detection performance, we found MPM had better performance than the EWMA, CUSUM methods. In theory, MPM method is simple, with fewer parameters and without the limit of the overall distribution of monitoring data. The results showed that the performance of MPM was stable under different test conditions, which indicated that the MPM method has a broad scope of application. These advantages prompted that this method should be first considered when designing an automatic disease surveillance system.

Consistent with previous evaluations of outbreak detection algorithms [5,34-36], the multiple linear regression results found that the ability to detect outbreaks

Table 5 Summary of Multiple Regression Analysis for Variables Predicting Sensitivity

\begin{tabular}{lllll}
\hline Variable & $\boldsymbol{\beta}$ & $\boldsymbol{S E}$ & $\boldsymbol{\beta}^{*}$ & $\boldsymbol{P}$-value \\
\hline Algorithms & & & & \\
CUSUM & 2.70 & 1.74 & 0.04 & 0.121 \\
MPM & 13.69 & 1.74 & 0.21 & $<0.001$ \\
EWMA(reference ) & & & & \\
Incubation & & & & \\
Short & -9.68 & 2.47 & -0.13 & $<0.001$ \\
Medium & -6.97 & 2.21 & -0.11 & 0.002 \\
Long(reference ) & & & & \\
Baseline counts & -12.01 & 1.38 & -0.20 & $<0.001$ \\
Outbreak magnitude & 16.11 & 0.66 & 0.55 & $<0.001$ \\
$\boldsymbol{R}^{\mathbf{2}=\mathbf{0 . 3 9}}$ & & & & \\
$\boldsymbol{F}=\mathbf{1 2 5 . 8 5}$ & & & & $<0.001$ \\
\hline $\boldsymbol{\beta}$ & & & &
\end{tabular}

$\beta^{*}$ standard regression coefficient.
Table 6 Summary of Multiple Regression Analysis for Variables Predicting Timeliness

\begin{tabular}{lllll}
\hline Variable & $\boldsymbol{\beta}$ & $\boldsymbol{S E}$ & $\boldsymbol{\beta}^{*}$ & $\boldsymbol{P}$-value \\
\hline Algorithms & & & & \\
CUSUM & 0.10 & 0.13 & 0.02 & 0.420 \\
MPM & -0.76 & 0.13 & -0.17 & $<0.001$ \\
EWMA(reference ) & & & & \\
Incubation & & & & \\
Short & -2.80 & 0.18 & -0.57 & $<0.001$ \\
Median & -2.42 & 0.16 & -0.56 & $<0.001$ \\
Long(reference ) & & & & \\
Baseline counts & 0.61 & 0.11 & 0.14 & $<0.001$ \\
$\begin{array}{l}\text { Outbreak magnitude } \\
\boldsymbol{R}^{\mathbf{2}}=\mathbf{0 . 2 8}\end{array}$ & -0.46 & 0.05 & -0.23 & $<0.001$ \\
$\boldsymbol{F}=\mathbf{7 3 . 2 9}$ & & & & \\
\hline $\boldsymbol{\beta}^{*}$ standard regression coefficient. & & & $<0.001$ \\
\hline
\end{tabular}

was better with lower baseline counts and larger magnitude. While previous studies inserted the actual counts with fixed outbreak case numbers, our study inserted the actual counts with outbreak case numbers based on the proportion of case distribution of simulated outbreaks.

Our study examined how different incubation periods affect the detection performance. There are three indicators(minimum, maximum and average of incubation period) to describe incubation, and these three indicators are closely related. To date there is still no definitely way of classifying the incubation period with these three indicators. So we tried the K-means clustering method to classify eight types of disease into three categories. The regression results showed that diseases of long incubation period had a higher sensitivity, but needed more time to detect than those of short and medium incubation period. Generally, the outbreaks of short incubation diseases occur ferociously and transiently, which are more easily to be missed by algorithms. The outbreaks of long incubation diseases, however, occur with longer duration, and can be detected by algorithms more accurately. As the early detection of outbreaks is important, additional work is still needed for timely detection long incubation diseases.

The biggest challenge for the evaluation of outbreak detection algorithms is to obtain a sufficiently large number of outbreak data with which to measure sensitivity and timeliness [33]. Injecting geometric shaped spikes into real surveillance data is a feasible approach [5,36-39]. In this study, in addition to inserting literatures-based simulated outbreak, we also used the public health emergency data. This method provided a solution on the issues where the simulated data may completely detach from real outbreaks and outbreaks in the real 
surveillance data may interfere with the performance evaluation.

We set a fixed false alarm rate of 0.05 , using simulation method to obtain the optimized parameters of different daily average counts data, and focused on the evaluation based on sensitivity and timeliness, which could make the comparison more clear.

There are several limitations to our study that should be taken into consideration. First, eight types of diseases clustering into three categories may not have a good representation. Second, the simulated outbreaks of eight diseases based on literature have some limitations to reflect the complexity of real outbreaks. Third, as only using two-year test datasets, we inserted a limited number of simulated outbreaks, which, to some extent, may affect the stability of the evaluation. In addition, due to the large amounts of computation in this study, we only compared three detection algorithms, so the evaluation of other algorithms needed to be further carried out.

\section{Conclusions}

The results of this study show that the MPM has better detection performance than EWMA, CUSUM. It can be considered as a prior algorithm for automatic infectious disease outbreak detection. Infectious disease outbreak detection performance varies with incubation period, baseline counts and outbreak magnitude. This suggests that the actual automatic infectious disease surveillance practice should take epidemic features into consideration, and select the appropriate algorithm to improve detection performance.

\section{Additional file}

Additional file 1 The optimized parameters for three algorithms, at an false alarm rate of $5 \%$.

\section{Competing interests}

The authors declare that they have no competing interests.

\section{Acknowledgements}

This research is funded by the Ministry of Science and Technology of China (2006BAK01A13, 2008BAI56B02, 2009ZX10004-201).

\section{Author details}

${ }^{1}$ Department of Occupational Health, West China School of Public Health, Sichuan University, 17 South Section 3 Renmin Road, Chengdu, Sichuan 610041, China. ${ }^{2}$ Key Laboratory of Surveillance and Early-warning on Infectious Disease, Chinese Center for Disease Control and Prevention (China CDC), 155 Changbai Road Changping District, Beijing, 102206, China.

\section{Authors' contributions}

JK and YJL designed the study, JK conducted the study and drafted the manuscript, WZY, DLZ and ZJL participated in the design of the study and involved in revising the manuscript. All authors have read and approved the final manuscript.
Received: 17 January 2012 Accepted: 8 June 2012

Published: 8 June 2012

\section{References}

1. Wang LD, Wang Y, Jin SG, Wu ZY, Chin DP, Koplan JP, Wilson ME: Emergence and control of infectious diseases in China. Lancet 2008, 372(9649):1598-1605.

2. Ministry of Health of the People's Republic of China: Emergency infectious disease prevention and control strategies. Beijing: Ministry of Health of the People's Republic of China; 2007.

3. Yang WZ, Li ZJ, Lan YJ, Wang JF, Ma JQ, Jin LM, Sun Q, Lv W, Lai SJ, Liao YL, Hu WB: A nationwide web-based automated system for outbreak early detection and rapid response in China. Western Pacific Surveillance and Response Journal 2011, 2(1):1-6.

4. Yang WZ, Lan YJ, Li ZJ, Ma JQ, Jin LM, Sun Q, LV W, Lai SJ: The application of national outbreak automatic detection and response system, China. Chin J Epidemiol 2010, 31(11):1240-1244.

5. Wang L, Ramoni MF, Mandl KD, Sebastiani P: Factors affecting automated syndromic surveillance. Artificial Intelligence in Medicine 2005, 34(3): 269-278.

6. Buckeridge DL: Outbreak detection through automated surveillance: $\mathrm{A}$ review of the determinants of detection. Journal of Biomedical Informatics 2007, 40(4):370-379.

7. Wang XL, Zeng $D$, Seale $H$, Cheng $H$, Luan RS, He X, Pang XH, Dou XF, Wang QY: Comparing early outbreak detection algorithms based on their optimized parameter values. Journal of Biomedical Informatics 2010, 43(1):97-103.

8. Pelecanos AM, Ryan PA, Gatton ML: Outbreak detection algorithms for seasonal disease data: a case study using ross river virus disease. $B M C$ Med Inform Decis Mak 2010, 10:74

9. Watkins RE, Eagleson S, Veenendaal B, Wright G, Plant AJ: Disease surveillance using a hidden Markov model. BMC Med Inform Decis Mak 2009, 9:39.

10. Jiang X, Cooper GF: A real-time temporal Bayesian architecture for event surveillance and its application to patient-specific multiple disease outbreak detection. Data Mining and Knowledge Discovery 2010, 20(3): 328-360.

11. Lu HM, Zeng D, Chen HC: Prospective Infectious Disease Outbreak Detection Using Markov Switching Models. leee Transactions on Knowledge and Data Engineering 2010, 22(4):565-577.

12. Shen Y, Cooper GF: A New Prior for Bayesian Anomaly Detection Application to Biosurveillance. Methods of Information in Medicine 2010 49(1):44-53

13. Alimadad A, Salibian-Barrera M: An Outlier-Robust Fit for Generalized Additive Models With Applications to Disease Outbreak Detection. Journal of the American Statistical Association 2011, 106(494):719-731.

14. Jiang FJ: Evaluation research on the quality of reporting in Emergency Public Reporting System. Master thesis. Chinese Center for Disease Control and Prevention:; 2006.

15. Stroup DF, Williamson GD, Herndon JL, Karon JM: Detection of aberrations in the occurrence of notifiable diseases surveillance data. Statistics in Medicine 1989, 8:323-329.

16. Farrington $C P$, Andrews $N J$, Beale $A D$, Catchpole MA: A statistical algorithm for the early detection of outbreaks of infectious disease. Journal of the Royal Statistical Society, Series A 1996, 159:547-563.

17. Williamson GD, Hudson GW: A monitoring system for detecting aberrations in public health surveillance reports. Statistics in Medicine 1999, 18:3283-3298.

18. Reis BY, Mandl KD: Time series modeling for syndromic surveillance. BMC Med Inform Decis Mak 2003, 3:2.

19. Zhang J, Tsui FC, Wagner MM, Hogan WR: Detection of Outbreaks from Time Series Data Using Wavelet Transform. AMIA Annu Symp Proc 2003:748-752.

20. Montgomery DC: Introduction to Statistical Quality Control. New York: John Wiley and Sons; 2001.

21. Hutwagner LC, Maloney EK, Bean NH, Slutsker L, Martin SM: Using laboratory-based surveillance data for prevention: an algorithm for detecting salmonella outbreaks. Emerg Infect Dis 1997, 3:395-400. 
22. Yang $W Z$, Xing $H X$, Wang $H Z$, Lan $Y J$, Sun $Q$, Hu SX, Lv W, Yuan ZA, Chen $Y X$, Dong $B Q: A$ study on early detection for seven infectious diseases. Chin J Epidemiol 2004, 25(12):1039-1041.

23. R Development Core Team: $R$ : A language and environment for statistical computing:; http://www.R-project.org.

24. Zhang RJ, Ji GH, Wang Y, Zai B: Investigation of a Shigella bacteria cause bacillary dysentery outbreak. Chin J Pest Control 2009, 25(6):437-438.

25. Li SX, Li LJ, Yu QF, Yu FB, Yu HX, Wu BW: Investigation of a scarlet fever outbreak in a school, Yuxi City. Chin J Sch Health 2005, 26(6):510-511.

26. Zhang $\Pi$, Chen $H X$ : Analysis of a mumps outbreak in a junior middle school of Huaxi town, Huayin City. Henan Journal of Preventive Medicine 2009, 20(6):449-450.

27. Huo HC: Report on a measles outbreak investigation. Disease surveillance 1997, 11:430.

28. Yao LJ, Chen HH: Analysis of a malaria outbreak investigation. Guangdong health and epidemic prevention 1998, 24(1):43-44.

29. Yin K, Li MQ, Liu JJ: Analysis of typhoid outbreak investigation in a school, Liuzhou. Modern preventive medicine 2007, 34(13):2559-2560.

30. Chen ZZ: A outbreak investigation of encephalitis B in 2000 year, Anxi County. Journal of Preventive Medicine Information 2001, 17(4):278-279.

31. Nong $Z M$, Meng $L$, Liu ZH, Jiang CH: Investigation of a hepatitis A outbreak in a school. Chin J Sch Health 2009, 30(3):274-275.

32. Ma YL: Infectious diseases. Shanghai: Shanghai scientific \& Technical Publishers; 2004

33. Wagner MM, Wallstrom G: Methods For Algorithm Evaluation. In Handbook of Biosurveillance. Edited by Wagner MM, Moore AW, Aryel RM. Burlington: Elsevier Press; 2006:301-310.

34. Nordin JD, Goodman MJ, Kulldorff M, Ritzwoller DP, Abrams AM, Kleinman K, Levitt MJ, Donahue J, Platt R: Simulated anthrax attacks and syndromic surveillance. Emerg Infect Dis 2005, 11(9):1396-1400.

35. Ozonoff A, Forsberg L, Bonetti M, Pagano M: Bivariate method for spatiotemporal syndromic surveillance. MMWR Morb Mortal Wkly Rep 2004, 53(Suppl):59-66.

36. Jackson ML, Baer A, Painter I, Duchin J: A simulation study comparing aberration detection algorithms for syndromic surveillance. BMC Med Inform Decis Mak 2000, 7:6.

37. Hutwagner L, Browne T, Seeman GM, Fleischauer AT: Comparing aberration detection methods with simulated data. Emerg Infect Dis 2005, 11:314-316.

38. Goldenberg A, Shmueli G, Caruana RA, Fienberg SE: Early statistical detection of anthrax outbreaks by tracking over-the-counter medication sales. Proc Natl Acad Sci USA 2002, 99(8):5237-5240.

39. Reis $B Y$, Pagano $M$, Mandl KD: Using temporal context to improve biosurveillance. Proc Natl Acad Sci USA 2003, 100(4):1961-1965.

\section{doi:10.1186/1471-2458-12-418}

Cite this article as: Kuang et al:: Epidemic features affecting the performance of outbreak detection algorithms. BMC Public Health 2012 12:418.

\section{Submit your next manuscript to BioMed Central and take full advantage of:}

- Convenient online submission

- Thorough peer review

- No space constraints or color figure charges

- Immediate publication on acceptance

- Inclusion in PubMed, CAS, Scopus and Google Scholar

- Research which is freely available for redistribution 